\title{
Educação alimentar e nutricional com universitários residentes de moradia estudantil
}

\author{
Food and nutritional education to residents university student housing
}

\author{
Priscila Bárbara Zanini Rosaa, Lisiane Giustib, Maurem Ramosc \\ a Nutricionista graduada pela Universidade Federal do Rio Grande do Sul (UFRGS). \\ a Acadêmica do Curso de Graduação em Nutrição da UFRGS. \\ b Nutricionista. Doutorado em Ciências da Saúde pela UFRGS. Professora adjunta do Departamento de Nutrição.
}

Objetivo: Identificar e ampliar os conhecimentos sobre alimentos para contribuir na mudança de hábitos e práticas alimentares inadequadas de adultos jovens residentes de moradia estudantil.

Materiais e Métodos: Estudo quase-experimental, no qual realizou-se uma intervenção educacional na área de alimentação e nutrição com 8 universitários residentes da casa do estudante. Foram realizados encontros semanais, durante três meses, na forma de diálogos e/ou atividades lúdicas e dinâmicas.

Resultados: Com os dados de um questionário sobre comportamento alimentar foi possível conhecer o comportamento alimentar dos participantes. A auto avaliação, realizada ao final da intervenção, mostrou que os mesmos incorporaram as orientações de alimentação saudável debatidas nos encontros em seu dia a dia e que se sentiam mais seguros na hora de comprar os alimentos. Pediu-se para que pontuassem de 0 a 10 alguns tópicos sobre a intervenção e a forma de condução dos encontros e obteve-se notas entre 9,4 e 10. Na dinâmica de "verdadeiro ou falso", em que se fez afirmativas que englobavam todos os assuntos abordados nos encontros, houve uma taxa de acerto das questões de 90 a 100\%.

Conclusão: Os encontros instigaram os universitários a mudarem alguns aspectos inadequados de sua alimentação e permitiram uma autonomia na hora de comprar alimentos (industrializados ou in natura).

Palavras-chave: hábitos alimentares; comportamento alimentar; saúde do adulto.

Objective: To identify and expand the knowledge about food in order to contribute to changing habits and inadequate feeding practices of young adults living in student houses.

Materials and Methods: Quasi-experimental study, in which an educational intervention in the area of food and nutrition with eight university students living in student houses was conducted. Weekly meetings were held for three months, in the form of dialogues and / or playful activities and dynamics.

Results: By using the data from a questionnaire on eating behavior it was possible to know the eating habits of the participants. The self-evaluation conducted at the end of the intervention showed that they incorporated the healthy eating guidelines discussed in the meetings in their daily lives and that they felt safer when buying food. They were asked to score from 0 to 10 some issues about the intervention and the guidance of the meetings, for which grades between 9.4 and 10 were obtained. In the dynamics of "true or false", in which statements regarding all topics covered in the meetings were performed, there was a hit rate of the questions between 90 and $100 \%$.

Conclusion: The meetings prompted the students to change some aspects of their inadequate food and allowed autonomy when buying food (industrialized or in kind).

Keywords: food habits; feeding behavior; adult health. 


\section{INTRODUÇÃO}

Segundo dados do Instituto Brasileiro de Geografia e Estatística (IBGE) ${ }^{1}$, o Brasil apresenta cerca de $12,5 \%$ de adultos do sexo masculino e $16,9 \%$ do sexo feminino com obesidade; além disso, 50,1\% dos homens e 48\% das mulheres possuem excesso de peso. A obesidade, doença crônica multifatorial caracterizada pelo acúmulo excessivo de gordura corporal2 é considerada um grande problema de saúde pública, uma vez que acarreta em inúmeras alterações metabólicas, como diabetes, doenças cardiovasculares e câncer, as quais aumentam a morbimortalidade dos indivíduos ${ }^{3}$.

De modo geral, obesidade está muito relacionada com uma dieta rica em gordura, açúcares e alimentos refinados (que concentram grande aporte energético) e, consequentemente, pobre em carboidratos complexos e fibras ${ }^{4}$. Concomitantemente, a redução da atividade física também é um fator contribuinte para o aumento de peso ${ }^{5}$. Dados extraídos da VIGITEL (2013) ${ }^{6}$ mostram que apenas $23,6 \%$ da população consome a ingestão diária de frutas e hortaliças recomendada pelo Ministério da Saúde e quase $50 \%$ da população é considerada insuficientemente ativa. Além disso, um aspecto muito importante, que interfere diretamente nas decisões cotidianas de alimentação e atividade física, é o comportamento alimentar. Entendese como comportamento alimentar os "procedimentos relacionados às práticas alimentares de grupos humanos (o que se come, quanto, como, quando, onde e com quem se come; a seleção de alimentos e os aspectos referentes ao preparo da comida) associados a atributos socioculturais" ${ }^{\prime 7}$.

Por isso, um dos papéis do nutricionista é o de ajudar as pessoas a modificar seus comportamentos alimentares, através da educação alimentar e nutricional para indivíduos e grupos populacionais ${ }^{8}$. O conhecimento sobre o que comer é um dos fatores que influencia na alimentação saudável. No entanto, a relação entre o que as pessoas sabem e o que as pessoas fazem, tem sido considerado como "altamente tênue". Sabe-se que o conhecimento não instiga a mudança, mas funciona como um instrumento quando as pessoas desejam mudar ${ }^{9}$.

A educação alimentar e nutricional visa promover a prática autônoma e voluntária de alimentação saudável, no contexto da realização do Direito Humano à Alimentação Adequada e da garantia da Segurança Alimentar e Nutricional ${ }^{10}$. É uma ação utilizada a fim de contribuir na prevenção da obesidade (que é tão prevalente nos adultos) e das doenças citadas anteriormente, bem como auxiliar nos seus tratamentos dietoterápicos.

Sabe-se que o meio universitário traz novas responsabilidades e demanda um grande tempo dos estudantes, fazendo com que haja sobreposição de atividades, mudanças comportamentais (principalmente alimentares) e outros fatores psicossociais envolvidos. Assim, pratos rápidos, sem horários pré-estabelecidos, e omissão de refeições são incorporados por esse tipo de população, o que leva a uma alimentação nutricionalmente inadequada ${ }^{11}$.

Sendo assim, o objetivo desse trabalho foi desenvolver uma intervenção educacional na área de alimentação e nutrição com um grupo de adultos jovens residentes na casa do estudante da Universidade Federal do Rio Grande do Sul (UFRGS). Buscou-se identificar e ampliar seus conhecimentos sobre os alimentos/alimentação e contribuir na mudança de hábitos e práticas alimentares inadequadas.

\section{MATERIAIS E MÉTODOS}

\section{Diagnóstico do grupo e planejamento das estratégias}

Trata-se de um estudo quasi-experimental com delineamento antes-depois. Através de cartazes de divulgação, os universitários moradores da casa do estudante da UFRGS foram convidados para participar dos encontros (amostragem por conveniência). Aceitaram o convite 8 universitários, com idade entre 20 e 26 anos, sendo 6 do sexo feminino e 2 do sexo masculino. Seis deles tinham índice de massa corporal (IMC) dentro da normalidade (eutróficos) e dois tinham sobrepeso, e estavam cursando diferentes graduações da UFRGS em Porto Alegre-RS. Realizaram-se encontros semanais num período de três meses, totalizando 11 encontros (1 para diagnóstico e planejamento e 10 para execução das estratégias), com duração de $1 \mathrm{~h}$, os quais ocorreram numa sala da Casa do Estudante Universitário da UFRGS (CEU-UFRGS).

A intervenção educacional fundamentou-se na teoria da dialogicidade ${ }^{12}$ descrita por Paulo Freire, considerando as características dos indivíduos, como questões culturais, nível de escolaridade e habilidades. Segundo este autor, o diálogo como fenômeno do ser humano, é ele mesmo, a palavra. Esta é constituída de duas dimensões: ação e reflexão, ou seja, práxis.

Desse modo, para iniciar o processo de diálogo, no primeiro encontro com o grupo (além de esclarecer como seriam conduzidos os encontros) iniciou-se o diagnóstico perguntando-Ihes as expectativas e dúvidas sobre alimentação de cada um dos participantes e aplicou-se um questionário sobre comportamento alimentar adaptado para o grupo em questão. Isso permitiu a investigação dos temas geradores, que seriam abordados e discutidos em cada encontro, e também a identificação do comportamento alimentar do grupo. 
Com os dados levantados no diagnóstico planejaram-se as estratégias para abordar os temas na forma da metodologia da conscientização ${ }^{13}$ descrita por Paulo Freire. Assim, traçouse os objetivos das ações e elaborou-se atividades lúdicas e participativas.

Foram seguidos os critérios éticos constantes da Resolução CNS/MS № 466/1214. A pesquisa foi aprovada pelo Comitê de Ética em Pesquisa, sob o número 11-0228. Após a aceitação em participar do grupo, foram apresentados os objetivos do estudo e solicitado aos participantes que, após a leitura e em caso de concordância, assinassem o termo de consentimento livre e esclarecido.

\section{Descrição das estratégias}

\section{Encontro 1}

O primeiro tema trabalhado foi a monotonia da dieta (isto é, comer sempre os mesmos alimentos). Ressaltaram-se os malefícios dessa conduta e as alternativas para solucionar este problema. Para isso, preparou-se um material com algumas orientações sobre planejamento das compras da semana (sugerindo que eles estipulassem um dia e um horário para as mesmas) e sobre planejamento do cardápio (sugerindo que experimentassem comprar alimentos variados para possibilitar maior diversificação das refeições). Neste dia levou-se ao encontro um suco verde para que eles pudessem provar algo que ainda não conheciam, com o intuito deles se permitirem experimentar um alimento novo sem preconceitos. Por fim, entregou-se algumas receitas de preparações rápidas e saudáveis (previamente debatidas com o grupo), que podem favorecer uma alimentação adequada, mesmo que os participantes tenham pouco tempo disponível para cozinhar. Para acompanhar a evolução dos participantes lançou-se um desafio: deveriam postar num grupo de uma mídia social comum utilizada para comunicação, uma foto de uma refeição preparada por eles que fosse diferente de sua rotina alimentar. Teve-se sucesso com esse desafio: a maioria postou e relatou ter mudado alguns aspectos da alimentação.

\section{Encontro 2}

Para abordar o segundo tema de interesse, realizouse uma atividade de mitos e verdades sobre alimentos e rótulos de produtos alimentares. Na dinâmica trabalhouse a quantidade de açúcar, sal e gordura dos alimentos industrializados utilizando potes que ilustram a quantidade desses ingredientes em determinados produtos. Eles foram separados em dois grupos e lhes foi entregue fichas com o nome do produto e quantidade de determinado ingrediente, assim eles tinham que apontar de qual frasco com açúcar, sal e gordura correspondia à quantidade descrita na ficha. Após, demos-lhes o gabarito, causando surpresa o resultado.
Em seguida fez-se um jogo sobre mitos e verdades sobre os rótulos, onde se fazia uma afirmação e, em grupo, eles tinham que levantar uma placa que estava escrito mito ou verdade. Por fim reforçou-se a importância de ler com atenção os rótulos com exemplos para eles compararem.

\section{Encontro 3}

Neste encontro trabalhou-se a temática alimentação à noite, principalmente antes de dormir, pois os participantes relataram que têm o hábito de dormir muito tarde e acabam ingerindo grande quantidade de alimentos nesse horário. Assim, conversou-se sobre quais alimentos devem ser evitados antes de dormir para que a digestão não seja lenta, e não prejudique o sono. Também, sugeriram-se alguns lanches que poderiam ser consumido pouco tempo antes de deitar que os ajudariam a se preparar para dormir.

\section{Encontro 4}

Aqui, a temática foi alimentação no pré e pós-exercício e suplementação proteica. Iniciou-se o diálogo esclarecendo o quanto os macronutrientes e micronutrientes são importantes para manutenção das funções do organismo (não apenas no exercício) e que momentos prolongados em jejum e dietas muito restritivas em carboidrato não são recomendados para indivíduos saudáveis, principalmente para praticantes de atividade física, pois prejudica a manutenção da glicemia e minimiza os estoques de glicogênio, causando um declínio no desempenho esportivo e na saúde do corpo humano ${ }^{15}$. Explicou-se que a refeição antes e depois do exercício deve ser balanceada em macronutrientes para fornecer energia durante o exercício e evitar perda de massa muscular ${ }^{15}$. Ressaltouse que a suplementação deve ser utilizada somente com orientação de um profissional especializado e apresentou-se um exemplo de dieta de um dia mostrando que o aporte de proteínas pode ser atingido somente com alimentos.

\section{Encontro 5}

Neste, abordaram-se as diferenças entre os açúcares, focando nos benefícios do açúcar mascavo e do açúcar demerara, visto que este não é muito conhecido. Foram trazidos os açúcares dentro de copinhos plásticos transparentes para eles visualizarem as diferenças de coloração e também experimentarem seus sabores. Além disso, esclareceu-se o que são adoçantes, quais os tipos que se encontra no mercado e quais os mais recomendados. $\mathrm{E}$ por fim, falou-se dos benefícios do mel.

\section{Encontro 6}

Leites, iogurtes e bebidas lácteas foram o tema do encontro 6. Para isso, inicialmente conversou-se sobre os leites oriundos 
tanto de vaca, quanto de cabra e ovelha. Explicou-se as perdas de nutrientes durantes os processamentos térmicos UHT e pasteurizado, falou-se da composição do leite, explicando os diferentes tipos (integral, semi-desnatado e desnatado) e também dos seus derivados. Após, realizou-se uma dinâmica: os participantes foram organizados em dois grupos e lhes foram entregues uma tabela nutricional em branco onde uma coluna estava escrito iogurte e na outra coluna escrito bebida láctea. Assim orientou-se que eles deviam preencher com um "+ " na coluna que eles achavam que tinha mais carboidrato ou mais proteína e assim por diante. Quando terminaram, mostrou-se o gabarito e debateu-se o porquê de tais diferenças. Também se comentou sobre os probióticos e explicou-se a diferença para os prebioticos. Ao fim, foi servida bebida láctea em um copo e iogurte em outro para sentirem a diferença de textura e sabor que apresentam.

\section{Encontro 7}

A temática deste encontro foi dietas da moda, alergias e intolerâncias alimentares. Conversou-se sobre intolerância a lactose, esclareceu-se como funciona a patologia e como é conduzida a dieta. Explicou-se que para quem não tem a intolerância não há evidências comprovando ser uma dieta saudável a ser seguida. A mesma coisa com o glúten: explicou-se a doença celíaca e debateu-se sobre a tendência de dietas "lacfree" e "glutenfree". Conversou-se também sobre dieta detox, dieta da proteína e quais as consequências que uma dieta muito restritiva pode causar ao organismo.

\section{Encontro 8}

Sucos, frutas e alimentos orgânicos foram o tema de discussão. Explicou-se as diferenças entre um produto orgânico e um convencional, falou-se das certificações, dos malefícios dos agrotóxicos e das inúmeras patologias que estão sendo estudadas devido ao consumo crônico desses produtos. Debateu-se a importância nutricional das frutas, das cores e quais compostos estão presentes em cada pigmento. Falou-se sobre a recomendação de consumo diário e quanto isso é benéfico. Além disso, conversou-se sobre sucos, diferenciando suco natural de néctar e suco em pó, alertando para terem cuidado com os rótulos que tentam enganar o consumidor. No início dessa conversa pediu-se que eles provassem um suco verde e adivinhassem quais ingredientes estavam presentes e só final foi revelado: cenoura, maçã, couve, laranja e hortelã, surpreendendo-os de como tantos ingredientes juntos podem formar um suco tão gostoso.

\section{Encontro 9}

Na sequência, o encontro em formato de roda de conversa abordou a temática: café, chás e chimarrão. Explicou-se os malefícios do consumo exagerado dessas bebidas, dos efeitos estimulantes que elas possuem e qual a dose recomendada por dia. Também se falou dos benefícios que alguns estudos relatam sobre o café e chimarrão e abordou-se um pouco sobre chás e seu uso na fitoterapia.

\section{Encontro 10}

No último encontro do grupo foi pedido aos participantes que realizassem uma auto avaliação e uma avaliação do projeto (tanto da forma de condução dos encontros quanto da relevância e conteúdo dos temas). Além disso, para avaliar os conhecimentos obtidos durantes os encontros, realizou-se uma atividade dinâmica de respostas "verdadeiras ou falsas". No fim desse encontro, os participantes em duplas trouxeram preparações que eles consideravam saudáveis (feitas por eles mesmos), através da troca de experiências com o grupo para fazer um encerramento comemorativo.

\section{Análise dos resultados}

Para análise dos dados foi utilizada a estatística descritiva, onde para o questionário de comportamento alimentar calculou-se a porcentagem de frequência das respostas; para a auto avaliação e a avaliação do projeto considerou-se a média de pontuação dada (0 a 10) para cada um dos tópicos questionados; e para a dinâmica de "verdadeiro ou falso" contabilizou-se quantos participantes acertavam as respostas, calculando-se a porcentagem de acertos, assim como suas justificativas.

\section{RESULTADOS}

Com os dados do questionário sobre comportamento alimentar obteve-se as seguintes informações: todos os estudantes saíram da casa dos pais para cursar a faculdade na UFRGS; todos moravam sozinhos no mínimo há um ano; todos achavam que comer frituras faz mal a saúde; todos consideravam alimentos saudáveis como os mais importantes pra saúde (arroz, feijão, frutas e leite); a maioria (90\%) não possuía tabus alimentares; grande parte $(75 \%)$ estava disposta a mudar a sua alimentação; metade consumia alimentos orgânicos por acharem mais saudável, já a outra metade desconhecia suas vantagens, não sabia onde comprar e achava caro; $60 \%$ liam os rótulos dos alimentos, mas não entendia bem o significado de cada item; $60 \%$ sabiam cozinhar alimentos integrais, mas possuíam preferência pelos refinados; grande parte $(90 \%)$ não comia mais frutas e verduras por falta de tempo e disposição de ir comprar, então só consumiam no restaurante universitário; nenhum deles era vegetariano; nenhum deixava de comer algum alimento por questões religiosas; todos gostavam de pão 
integral, embora consumissem mais o pão branco; $40 \%$ eram viciados em doces e gostavam de "beliscar"; $75 \%$ não achavam importante comer alimentos que lembrem a infância, entretanto 50\% preparavam comidas típicas da origem da família; $90 \%$ consumiam alimentos trazidos pelo grupo de amigos; a maioria (75\%) achava que não são só as mulheres que devem cozinhar; todos consumiam arroz e feijão pelo menos cinco vezes na semana; $60 \%$ praticavam atividade física; e a maioria (75\%) não conseguia seguir recomendações de alimentação saudável vistas na mídia.

Para realização de uma autoavaliação, ao final da intervenção, pediu-se para que pontuassem de 0 a 10 os seguintes tópicos (entre parênteses está descrita a média obtida): sinto-me mais seguro para comprar alimentos in natura ou industrializados $(8,5)$, incorporei as orientações abordadas nos encontros no meu dia a dia $(8,8)$, conversei e/ou expliquei brevemente a um amigo/familiar sobre algo que aprendi nos encontros (8), o projeto me forneceu informações importantes que motivaram a mudar minha alimentação $(9,4)$, o projeto me fez entender o papel da alimentação na saúde humana $(9,5)$.

Sobre a intervenção e a forma de condução dos encontros pediu-se para que pontuassem de 0 a 10 os seguintes tópicos (entre parênteses será descrito a média obtida): criatividade nas atividades didáticas (10), forma de exemplificação de fácil entendimento $(9,9)$, forma de condução dos encontros $(9,6)$, relacionamento das autoras com os participantes (10), disponibilização de materiais extra $(9,4)$, relevância dos materiais disponibilizados $(9,5)$, os temas abordados atingiram minhas expectativas $(9,4)$.

Na dinâmica de respostas de verdadeiro ou falso faziamse afirmativas que englobavam todos os assuntos abordados nos 10 encontros e individualmente eles deveriam levantar uma placa de verdadeiro ou falso e justificar por quê. Obteve-se uma taxa de acerto das questões de 90 a 100\%, bem como as justificativas, que foram $100 \%$ corretas.

\section{DISCUSSÃO}

Pelos resultados obtidos, acredita-se que o estudo conseguiu identificar e ampliar os conhecimentos sobre os alimentos/alimentação e contribuiu na mudança de alguns hábitos e práticas alimentares inadequadas dos participantes. Sendo assim, o objetivo do trabalho foi alcançado.

Através do questionário de comportamento alimentar, percebe-se que os estudantes possuem uma noção básica sobre alimentação saudável, por exemplo, quando relatam que comer frituras faz mal a saúde ou quando metade deles diz consumir produtos orgânicos. Entretanto, verificam-se alguns comportamentos inadequados como preferir alimentos refinados aos integrais, beliscar alimentos fora de hora, consumir alimentos ricos em açúcar e não consumir a quantidade satisfatória de frutas e verduras por falta de tempo e disposição. Segundo alguns autores ${ }^{16,17,18}$, isso ocorre, muitas vezes, devido aos estudantes terem um estilo de vida estressante e com uma carga horária grande a cumprir, levando-os a adotarem uma alimentação não saudável (caracterizado por omissão de refeições e lanches e pelo consumo de alimentos altamente processados ricos em gordura saturada, açúcar e/ou sódio) e a terem um hábito sedentário, o que pode os tornar suscetíveis a desenvolver obesidade.

Para Alves e Boog ${ }^{19}$, o insucesso em cuidar da própria alimentação está relacionado ao insucesso no cuidado consigo mesmo, pois esse cuidado demanda e disputa tempo e dedicação com a necessidade que o estudante tem de incorporar-se e vivenciar a própria universidade. Isso indica que o cuidado com a alimentação não é visto como prioridade para o estudante, pois sua atenção está voltada, principalmente, ao desempenho acadêmico, a sua rede de relacionamentos e a vida cultural que a universidade potencialmente propicia, isto é, está mais preocupado em "aproveitar" a vida universitária.

Com os resultados da autoavaliação dos participantes, acredita-se que os encontros influenciaram de maneira positiva na alimentação do grupo, auxiliando-os na compra de alimentos e fazendo com que eles transmitissem um pouco do que aprenderam ao próximo. Isso demonstra uma mudança de comportamento em relação à alimentação saudável. Com a avaliação pelos universitários do modo de condução da intervenção, considera-se que os resultados obtidos foram muito positivos, mostrando que eles gostaram da abordagem dos temas, da condução dos encontros e das atividades dinâmicas. E ainda, com a dinâmica de verdadeiro ou falso vê-se, mais uma vez, que os estudantes aprenderam de fato o que foi debatido nos encontros e que a forma de abordagem dos temas foi eficaz.

Um estudo com intervenção nutricional de forma dinâmica realizado por Teixeira et al. ${ }^{20}$, com um grupo de mulheres adultas tendo duração de dois meses, apresentou mudanças alimentares significativas, como a redução do consumo de gorduras e o aumento do consumo de frutas, verduras e legumes. Felippe et al. ${ }^{21}$ buscando avaliar a qualidade alimentar de grupos de adultos que passaram ou não por um processo de reeducação alimentar verificou que, após 18 meses de intervenção, aqueles que foram expostos ao processo tinham uma melhor qualidade da dieta em relação ao grupo não exposto, com resultados positivos para o consumo de diversos grupos alimentares, entre eles frutas e verduras, laticínios, óleos e doces. Esses estudos mostram 
que a intervenção nutricional educativa de caráter prático, produz efeitos importantes na mudança de comportamento alimentar, assim como encontrado no presente trabalho.

A construção do conhecimento que favoreceu as melhorias alimentares observadas nesse estudo é considerada um passo inicial de mudanças efetivas e duradouras de hábitos alimentares e de vida. Alves e Boog ${ }^{19}$ ressaltam que o comportamento coletivo pode ser um fator importante para melhoria da qualidade da alimentação e da própria convivência entre os membros de um grupo. Tendo em vista que a casa do estudante é um meio privilegiado de integração social, isso pode explicar os resultados positivos obtidos com a intervenção do presente estudo e também pode auxiliar no processo de continuidade dessa mudança de comportamento.

Com este trabalho, pode-se perceber que ainda faltam referências de como trabalhar educação alimentar entre universitários moradores de Casa do Estudante, bem como para outros tipos de população. Também, há escassez de estudos que realizam intervenções nutricionais com estudantes. Além disso, é necessário mais conhecimento teórico e prático para trabalhar temáticas de comportamento alimentar para identificar os aspectos que interferem na alimentação desses universitários. Por fim, percebe-se que há necessidade de programas de educação alimentar e nutricional especifico para grupos com suas respectivas características, pois não se podem trabalhar as mesmas estratégias com qualquer público.

\section{REFERÊNCIAS}

1. Instituto Brasileiro de Geografia e Estatística. Pesquisa de Orçamentos Familiares 2008-2009 - POF. Antropometria e Estado Nutricional de Crianças, Adolescentes e Adultos no Brasil. Rio de Janeiro: IBGE; 2010.

2. World Health Organization. Obesity: Preventing and managing the global epidemic. Report of a WHO Consultation on Obesity. Geneva: WHO; 1998.

3. World Health Organization. Obesity: preventing and managing the global epidemic. Report of a WHO consultation on obesity. Geneva: $\mathrm{WHO} ; 2000$.

4. Instituto Brasileiro de Geografia e Estatística. Pesquisa de Orçamentos Familiares 2008-2009 - POF. Pesquisa de Orçamentos Familiares 2008-2009: análise do consumo alimentar pessoal no Brasil. Rio de Janeiro: IBGE; 2011.

5. Mendonça CP, Anjos LA. Aspectos das práticas alimentares e da atividade física como determinantes do crescimento do sobrepeso/ obesidade no Brasil. Cad. Saúde Pública. 2004;20(3):698-709. http://dx.doi.org/10.1590/S0102-311X2004000300006

6. Brasil. Secretaria de Vigilância em Saúde. Secretaria de Gestão Estratégica e Participativa. Vigitel Brasil 2012: vigilância de fatores de risco e proteção para doenças crônicas por inquérito telefônico. Brasília: Ministério da Saúde; 2013.
7. Ramos M, Stein LM. Desenvolvimento do comportamento alimentar infantil. J Pediatr. 2000;76(3):S229-37. http://dx.doi.org/10.2223/ JPED.160

8. Diez RWG. Representações Sociais da Comida no Meio Urbano: algumas considerações para o estudo dos aspectos simbólicos da alimentação. Rev Cad Debate. 1994;2:12-24.

9. Chapman KM, Ham JO, Liesen P, Winter L. Appeying behavioral models to dietary education of eldery diabetic patients. J Nutr Educ Behav.1995;27(2):75-9. http://dx.doi.org/10.1016/S00223182(12)80345-1

10. Brasil. Ministério do Desenvolvimento Social e Combate à Fome. Educação Alimentar e Nutricional: o direito humano a alimentação adequada e o fortalecimento de vínculos familiares nos serviços socioassistenciais. Brasília: Ministério do Desenvolvimento Social e Combate à Fome; 2014.

11. Cota RP, Miranda LS. Associação entre constipação intestinal e estilo de vida em estudantes universitários. Rev Bras Nutr Clin. 2006;21(4):296-301.

12. Freire P. Pedagogia do oprimido. Rio de Janeiro: Paz e Terra; 2005.

13. Freitas ALS. Pedagogia da Conscientização: um legado de Paulo Freire à formação de professores. Porto Alegre: Edipucrs; 2004.

14. Brasil. Ministério da Saúde. Resolução no 466, de 12 de dezembro de 2012. Normas da ética na pesquisa com seres humanos. DOU. 2013 Jun 13:59.

15. Ormsbee MJ, Bach CW, Baur DA. Pre-Exercise Nutrition: The Role of Macronutrients, Modified Starches and Supplements on Metabolism and Endurance Performance. Nutrients. 2014;6(5):1782-808. http://dx.doi.org/10.3390/nu6051782

16. Fernandes J, Arts J, Dimond E, Hirshberg S, Lofgren IE. Dietary factors are associated with coronary heart disease risk factors in college students. Nutr Res. 2013;33(8):647-52. http://dx.doi.org/10.1016/j. nutres.2013.05.013

17. Neslisah R, Emine AY. Energy and nutrient intake and food patterns among Turkish university students. Nutr Res Pract. 2011;5(2): 117-23. http://dx.doi.org/10.4162/nrp.2011.5.2.117

18. Nelson MC, Story M, Larson NI, Neumark-Sztainer D, Lytle LA. Emerging adulthood and college-aged youth: an overlooked age for weight-related behavior change. Obesity (Silver Spring). 2008;16(10):2205-11. http://dx.doi.org/10.1038/oby. 2008. 365

19. Alves HJ, Boog MAF. Comportamento alimentar em moradia estudantil: um espaço para a promoção da saúde. Rev Saúde Públ. 2007;41(2):197-204. http://dx.doi.org/10.1590/S003489102007000200005

20. Teixeira PDS, Reis BZ, Vieira DAS, Costa D, Costa JO, Raposo OFF, Wartha ERSA, Netto RSM. Intervenção nutricional educativa como ferramenta eficaz para mudança de hábitos alimentares e peso corporal entre praticantes de atividade física. Ciênc Saúde Coletiva. 2013;18(2):347-56. http://dx.doi.org/10.1590/S141381232013000200006

21. Felippe F, Balestrin I, Silva FM, Schneider AP. Qualidade da dieta de indivíduos expostos e não expostos a um programa de reeducação alimentar. Rev Nutr. 2011;24(6):833-44. http://dx.doi.org/10.1590/ S1415-52732011000600004 